\title{
A Study of Correlation between Abnormal Glucose Tolerance (AGT), Insulin Resistance (IR) and Abdominal Circumference (AC) in Young Girls with Polycystic Ovarian Syndrome at a Multi-Specialty Hospital in South Kolkata
}

\author{
Shubhasis Banerjee ${ }^{1}$, Lipika Das Mukhopadhyay², Susmita Basu³
}

${ }^{1}$ Department of Obstetrics and Gynaecology, Sri Aurobindo Seva Kendra (EEDF Hospital), Kolkata, West Bengal, India. ${ }^{2}$ Department of Obstetrics and Gynaecology, KPC Medical College \& Hospital, Kolkata,

West Bengal, India. ${ }^{3}$ Calcutta National Medical College \& Hospital, Kolkata, West Bengal, India.

\section{ABSTRACT}

\section{BACKGROUND}

Polycystic ovarian syndrome is an array of metabolic, hormonal and reproductive dysfunction that affects women. It is an important cause of female infertility and also leads to other medical conditions including anxiety, depression, obesity, endometrial cancer, liver disease, cardio-vascular disease and type 2 diabetes mellitus which results from insulin resistance. Compared to the others, these patients may significantly develop low quality of life related to health.

\section{METHODS}

The current study analyses the data, retrospectively of female patients, in the age range of 16 - 28 years, who presented at the out-patient clinic of gynaecology \& obstetrics, in a tertiary care multi-specialty hospital at South Kolkata. Data was collected over a six months period and was analysed using appropriate statistical method.

\section{RESULTS}

A total of fifty-seven cases was included in the study with a median age of 22 years and a median BMI of $24 \mathrm{Kg} / \mathrm{m}^{2}$ (range 18 - 36). Insulin resistance was found in 25 patients (43.86\%), and impaired glucose tolerance was found in 6 patients (10.53 $\%$ ). All patients of frank diabetes (post-prandial glucose $>140 \mathrm{mg} \%$ ) were suffering from insulin resistance. Abdominal circumference was above the cut-off level $(>80$ $\mathrm{cm})$ in 28 patients (49.12\%).

\section{CONCLUSIONS}

From the current study, it is established that impaired glucose tolerance alone cannot be a good indicator of body's disturbed glucose metabolism and there is increased need to measure insulin resistance to diagnose prospective diabetic condition associated with PCOS.

\section{KEY WORDS}

Abnormal Glucose Tolerance, Insulin Resistance, PCOS, Polycystic Ovarian Syndrome
Corresponding Author: Dr. Shubhasis Banerjee, Department of Obstetrics \& Gynaecology, SASK (EEDF Hospital), 1H, Gariahat Road, Kolkata - 700068, West Bengal, India.

E-mail: banerjeecalcutta@gmail.com

DOI: $10.14260 /$ jemds/2020/828

How to Cite This Article:

Banerjee S, Mukhopadhyay LD, Basu S. A study of correlation between abnormal glucose tolerance (AGT), insulin resistance (IR) and abdominal circumference (AC) in young girls with polycystic ovarian syndrome at a multi-specialty hospital in South Kolkata. J Evolution Med Dent Sci 2020;9(50):3773-3777, $10.14260 /$ jemds $/ 2020 / 828$

Submission 31-07-2020,

Peer Review 25-10-2020,

Acceptance 03-11-2020,

Published 14-12-2020.

Copyright (C) 2020 Shubhasis Banerjee et al. This is an open access article distributed under Creative Commons Attribution License [Attribution 4.0 International (CC BY 4.0)] 


\section{BACKGROUND}

PCOS / PCOD (Poly-Cystic Ovarian Syndrome / Disorder) is a gynaecological disorder comprising of secondary amenorrhoea, hirsutism (with or without acne), obesity and ovaries with multiple small cysts (volume of one or both ovaries > $10 \mathrm{cc}$ by USG). ${ }^{1}$ It is one of the most common human endocrinopathies affecting 12-21\% of woman of reproductive age group. Up to $70 \%$ of women with PCOS remain undiagnosed. The ovaries develop multiple tiny small cystic collections containing fluidic substance and may completely or partially fail to release ovums which may lead to infertility, primary or secondary.$^{1}$

Though the exact cause behind PCOS is unknown, but there are evidences which suggest that it may involve a combination of endocrine as well as genetic factors. In the past, it was found in numerous studies, repeatedly, that polycystic patients harbour elevated risk for conditions like hypertension, heart disease, dyslipidaemia, type 2 diabetes mellitus and other significant metabolic ordeal linked to insulin resistance.2,3,4 These patients develop lower QOL (Quality of Life) ${ }^{5,6}$ in terms of health, and are at higher risk for mental disorders like depression. ${ }^{7}$ Spitzer et al demonstrated overall occurrence of depressive spells as high as $12 \%$ in a significantly large multicentric study of OBG patients. ${ }^{8}$ Women with PCOS have a higher risk of cardiovascular sequelae, especially indigenous women who are already at increased baseline risk. Management of polycystic ovarian syndrome involves treatment of current conditions, as well as prevention of complications arising from it including frank diabetes. Thus, treatment of PCOS should include multi-disciplinary approach to provide management of psychological and fertility issues.

There is a range of symptoms in PCOS that women can experience depending on the age of the presentation. In younger women, reproductive symptoms predominate; whereas metabolic features are prevalent in older women. The onset of symptoms can be triggered by environmental factors and genetic predisposition. Ethnicity may also have impact over PCOS as some population groups have higher risk of PCOS; as for example, South-East Asian women are less likely to have hirsutism.9,10.

Attie et al. ${ }^{11}$ established in a study that obesity associated with chronic inflammation triggers the action of adipocytokines which in turn may contribute to insulin resistance (IR). It was found that adipokines play an important role in paracrine and autocrine signalling. The liver communicates with adipose tissue through adipokines, lipoproteins and lipid particles.

Insulin resistance and abnormal glucose tolerance is prevalent in patients of PCOS in varied degrees. ${ }^{12}$ Therefore, a screening Oral Glucose Tolerance Test (OGTT) along with serum insulin level test is highly recommended independent of the obesity pattern of PCOS patients.

During initial assessment of women for PCOS, few points to be considered which are further addressed in the guideline. ${ }^{13}$ After menarche, it may take up to 2 years for the menstrual cycle to be regular. Irregular cycles persisting into the third-year post-menarche should be investigated for PCOS. Ultrasound is not a reliable diagnostic tool in the diagnosis of PCOS in adolescent and young women, as up to $70 \%$ of young women may have polycystic ovaries on ultrasound. ${ }^{14}$ Initial investigations must exclude other causes of same presentation. Hormonal assessment should consider thyroid function tests, serum prolactin and follicle stimulating hormone (FSH) levels.

\section{METHODS}

The current study is representing analysis of data, retrospectively, on female patients, suffering from PCOS, aging between 16 - 28 years who attended in the Out-PatientDepartment of Gynaecology \& Obstetrics, in a tertiary care multi-specialty hospital at South Kolkata, over six months periods ( $6^{\text {th }}$ October' 2019 - $5^{\text {th }}$ March 2020). PCOS was diagnosed using Rotterdam criteria. (2003). ${ }^{15,16,17}$ The patients were assessed by clinical and psychological examination and evaluation of hormonal levels as advised, to diagnose PCOS according to the criteria which requires the presence of the two of the following: oligo / anovulation, hyperandrogenism (Clinical-hirsutism or less commonly male pattern alopecia or Biochemical-raised FAI or testosterone), or polycystic ovaries on ultrasound. Oligo / anovulation is usually seen in women with menstrual cycle greater than 35 days apart or conversely with shorter cycle ( $<21$ days); even a woman with regular cycle may be anovulatory. Ovulatory status is best diagnosed with luteal phase progesterone assessment. Hyperandrogenaemia is best diagnosed with free testosterone or Free Androgen Index (FAI). Polycystic ovaries on ultrasound scan are diagnosed when at least 10 small antral follicles are seen in each ovary. A unilateral polycystic ovary is equally significant.

Conventionally, BMI (Body Mass Index) ${ }^{18}$ is defined as a ratio of weight in $\mathrm{Kg}$ to height in $\mathrm{m}^{2}$. Abdominal circumference was measured with a metric tape (twice, to get an average). Care was taken to avoid errors in reading by asking the subjects to wear a light cloth during measurement. As recommended by Legro et al, ${ }^{19}$ abdominal circumference is measured at the maximum circumference between lower margin of the $10^{\text {th }} \mathrm{rib}$ and the highest point of iliac crest. Obesity is defined when BMI is $\geq 23 \mathrm{~kg} / \mathrm{m}^{2}$. An abdominal circumference of $\geq 80 \mathrm{~cm}$ (32 inch) was also considered as above cut-off level.20,21 Hirsutism was assessed by $\mathrm{mFG}$ score. ${ }^{22}$ Nine different body places were observed for hair growth pattern, hirsutism is considered if $\mathrm{mFG}$ score $>9$. To know the follicular status of ovary, ultrasound study was done on patients. Married women underwent Trans-Vaginal Sonography (TVS), whereas Trans-Abdominal Sonography (TAS) was preferred in girls who were not married yet. Few biochemical assessments were also included in the study, e.g. serum testosterone level (electro-chemiluminescence method), insulin level (Elecsys 2010, Roche), and serum glucose level ( 2 hours after meal). Hormonal tests are recommended to be done on the second or the third day of an induced bleeding. AGT screening is based on oral glucose tolerance test and WHO criteria using 75 gm oral glucose intake followed by measurement of plasma glucose and insulin after 2 hours. ${ }^{23}$ Insulin resistance was diagnosed as a ratio of Post-Prandial Blood Sugar (PPBS): Post-Prandial Insulin (PPI). Conventionally, IR is defined when the ratio is $\leq 1.0 . .^{24}$

In this study, post-prandial blood glucose estimation is preferred over measuring fasting glucose, because of two 
reasons. Firstly, it is easier to diagnose impaired glucose tolerance if challenged with specific amount of glucose before performing the test. Secondly, by measuring post-prandial insulin and making a ratio of PPBS: PPI (the insulin resistance), one can get clear idea about body's ability to neutralize glucose after each meal.

Written consent was taken from all the participants of the study. They were enlightened regarding the goal of the study. Patients were informed that there would be no requirement of any extra tests or procedures.

Permission was obtained from the Ethical Committee of the multi-specialty hospital where the first author conducted the study. All procedures carried out, followed the revised Helsinki declaration-200825 for the ethical standards to be maintained for research on mankind.

\section{Statistical Analysis}

Statistical analysis of collected data was done using SPSS software (version 13.0, IBM Corporation). Inter-group comparison was done using Chi-square test. A p-value $<0.05$ was considered as significant in terms of analysis.

\section{RESULTS}

A total of fifty-seven cases were included in this study with a median age of 22 years (range 16 - 28 years); a median BMI of $24 \mathrm{~kg} / \mathrm{m}^{2}$ (range 18 - 36). Insulin resistance was found in 25 / 57 patients (43.86\%), and impaired glucose tolerance was found in 6 / 57 patients $(10.53 \%)$.

\begin{tabular}{|c|c|c|c|c|c|c|}
\hline & & $\begin{array}{c}\text { IR Present } \\
\text { (\%) }\end{array}$ & $\begin{array}{c}\text { IR } \\
\text { Absent } \\
(\%)\end{array}$ & $\begin{array}{c}\text { Signifi- } \\
\text { cance }\end{array}$ & $\begin{array}{c}\text { OR } \\
(95 \% \\
\text { CI })\end{array}$ & $\begin{array}{c}\text { AOR } \\
(95 \% \\
\text { CI })\end{array}$ \\
\hline \multirow{2}{*}{$\begin{array}{c}\text { Abdominal } \\
\text { Circumference }\end{array}$} & Obese & $14(50.0)$ & $14(50.0)$ & \multirow{2}{*}{$\begin{array}{c}\mathrm{X}^{2}=0.843 \\
\mathrm{dF}=1 \\
\mathrm{p}=0.358\end{array}$} & \multirow{2}{*}{$\begin{array}{c}1.636 \\
(0.570- \\
4.696)\end{array}$} & \multirow{2}{*}{$\begin{array}{r}1.187 \\
(0.339- \\
4.154)\end{array}$} \\
\hline & Normal & $11(37.9)$ & $18(62.1)$ & & & \\
\hline \multirow{2}{*}{$\begin{array}{l}\text { Blood Glucose } \\
\text { Level }\end{array}$} & IGT & $5(20.0)$ & $20(80.0)$ & $X^{2}=0.2 .641$ & 7.750 & 7.619 \\
\hline & NGT & $1(3.12)$ & $\begin{array}{c}31 \\
(96.88)\end{array}$ & $\begin{array}{c}\mathrm{dF}=1 \\
\mathrm{p}=0.104\end{array}$ & $\begin{array}{l}(0.842- \\
71.317)\end{array}$ & $\begin{array}{c}0.693- \\
83.793)\end{array}$ \\
\hline \multicolumn{7}{|c|}{ able 1. Factors Affecting Insulin Resistance (IR) } \\
\hline
\end{tabular}
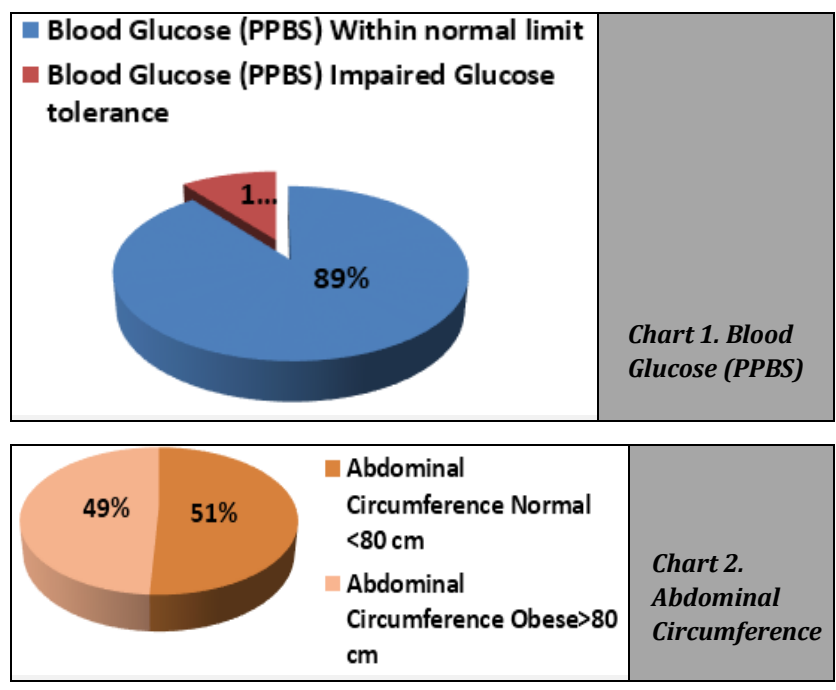

All patients of frank diabetes (post-prandial glucose $>140$ mg \%) were suffering from insulin resistance. Abdominal circumference was above cut-off level $(>80 \mathrm{~cm})$ in $28 / 57$ patients (49.12\%).

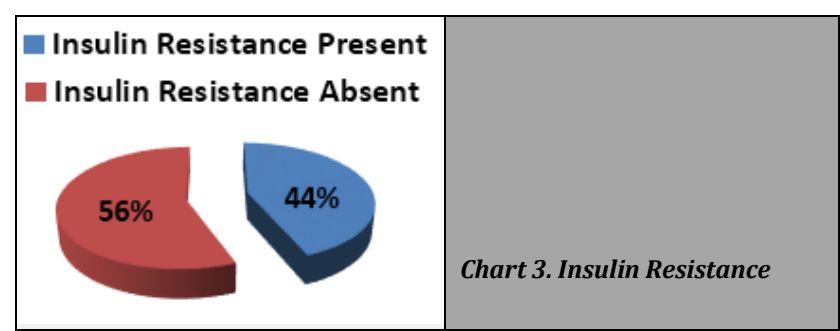

DISCUSSION

The majority of PCOS patients suffer from insulin resistance. Their body needs higher insulin level to titrate the same amount of glucose comparing a normal person. It is a symptom of PCOS and at the same time, it is the most powerful physiological reason to cause the array of disorders. Thus, testing for insulin resistance helps to differentiate it from other similar conditions like PCOS. ${ }^{26}$

Insulin resistance may be observed in both obese and nonobese PCOS patients. By observing insulin resistance, one can differentiate patients who lack an insulin related symptom; as for example, patients with adrenal PCOS 27 and a considerably large group of patients having amenorrhoea because of disturbed hypothalamus-pituitary axis but have wrongly diagnosed as 'lean PCOS'. There is evidence from research that demonstrates an increasing rate of PCOS correlated with weight gain, over previous 10 years ${ }^{28}$. Another study portrays a proportionate increase of polycystic disorder to rising occurrence of type-II diabetes. ${ }^{29}$

Conventional treatment strategy for insulin resistance includes exercise, weight loss and medications like metformin which improves insulin sensitivity at cellular level. ${ }^{30}$ It is observed that resistance training may also be effective in few cases. ${ }^{31}$ Combined OCPs (Oral Contraceptive Pills) are among other drugs that is being widely used in the treatment of PCOS, but studies revealed that this drugs can alter body's usual response to blood glucose and can actually induce insulin resistance which is the major cause of PCOS. ${ }^{32}$

Increased dietary intake of fructose also contributes to insulin resistance. Though low amount of fructose from fruits does not induce insulin resistance, but substantially higher content of fructose in junk foods has a more adverse effect. It is obvious that body's metabolic response to higher and lower quantity of sugar is very different. ${ }^{33}$

Subclinical deficiency of magnesium and inositol may contribute to insulin resistance. A meta-analytical study, conducted in 2018, which included ten different RCTs (Randomized Controlled Trials), proved that inositol has some certain role in improvement of insulin resistance which means gross improvement in symptoms like ovulation dysfunction, irregular menstrual cycles, hormonal misbalance and mental health. ${ }^{34}$ Another study revealed that supplementation with trace minerals like $\mathrm{Mg}$ has some positive effect on symptomatic relief of polycystic disease. ${ }^{35}$

Delayed menstruation is a sign of altered ovarian activity. ${ }^{36,37}$ Traditionally, PCOS is one of the causative factors for type 2 diabetes. Insulin resistance is considered an important problem in the pathogenesis of PCOS. 
Our study reveals, there are non-obese PCOS women who demonstrated impaired glucose tolerance and deranged insulin levels though having normal fasting sugar levels. Those patients had not developed frank diabetes, but at increased risk of developing diabetes in later life. Thus, they may get benefited from serial monitoring of blood glucose and metformin therapy. Findings of this study are in line with other studies of regarding PCOS. All of these study demonstrated that results of impaired glucose tolerance tests were not adequate to establish risks associated with metabolism. ${ }^{2-5,23}$ In this study, it is found, abdominal obesity contributed to higher post-prandial insulin level, which is consistent with prior literatures. ${ }^{38}$ We have found higher PPI levels in the high BMI group which is consistent with prior studies. ${ }^{39}$

\section{CONCLUSIONS}

There have been reports of conversion of NGT (Normal Glucose Tolerance) to AGT and subsequently, AGT to T2DM (Type 2 Diabetes Mellitus) in population with PCOS as well as in healthy subjects. Results of this study showed, post-prandial insulin levels to be increased in abnormal glucose tolerant (AGT) patients in comparison to (NGT) patients. In PCOS patients, it is difficult to find out prospective frank T2DM without measuring serum insulin level and comparing them to insulin resistance. Insulin resistance is an integral part of most patients in PCOS. The overall risk of conversion from NGT to AGT or T2DM in women with PCOS is higher in the presence of obesity.

Data sharing statement provided by the authors is available with the full text of this article at jemds.com.

Financial or other competing interests: None.

Disclosure forms provided by the authors are available with the full text of this article at jemds.com.

We are grateful to all of the patients who took part in this study and thankful to the administrative and clinical staff of the Aurobindo Seva Kendra (EEDF Hospital). We express our gratitude to Dr. (Prof.) Sudhindra Mohan Bhattyacharya (KPC Medical College \& Hospital, Kolkata) for his valuable opinion.

\section{REFERENCES}

[1] Sheehan MT. Polycystic ovarian syndrome: diagnosis and management. Clin Med Res 2004;2(1):13-27.

[2] Galluzzo A, Amato MC, Giordano C. Insulin resistance and polycystic ovary syndrome. Nutr Metab Cardiovasc Dis 2008;18(7):511-8.

[3] Yang G, Li L, Chen W, et al. Circulating preptin levels in normal, impaired glucose tolerance, and type 2 diabetic subjects. Ann Med 2009;41(1):52-6.

[4] Legro RS, Kunselman AR, Dodson WC, et al. Prevalence and predictors of risk for type 2 diabetes mellitus and impaired glucose tolerance in polycystic ovary syndrome: a prospective, controlled study in 254 affected women. J Clin Endocrinol Metab 1999;84(1):165-9.

[5] Elsenbruch S, Hahn S, Kowalsky D, et al. Quality of life, psychosocial well-being, and sexual satisfaction in women with polycystic ovary syndrome. J Clin Endocrinol Metab 2003;88(12):5801-7.
[6] McCook JG, Reame NE, Thatcher SS. Health-related quality of life issues in women with polycystic ovary syndrome. J Obstet Gynecol Neonatal Nurs 2005;34(1):12-20.

[7] Hollinrake E, Abreu A, Maifeld M, et al. Increased risk of depressive disorders in women with polycystic ovary syndrome. Fertil Steril 2007;87(6):1369-76.

[8] Spitzer RL, Williams JB, Kroenke K, et al. Validity and utility of the PRIME-MD patient health questionnaire in assessment of 3000 obstetric - gynecologic patients: the PRIME-MD Patient Health Questionnaire ObstetricGynecology Study. Am J Obstet Gynecol 2000;183(3):75969.

[9] March WA, Moore VM, Willson KJ, et al. The prevalence of polycystic ovary syndrome in a community sample assessed under contrasting diagnostic criteria. Hum Reprod 2010;25(2):544-51.

[10] Boyle JA, Cunningham J, O'Dea K, et al. Prevalence of polycystic ovary syndrome in a sample of Indigenous women in Darwin, Australia. Med J Aust 2012;196(1):626.

[11] Attie AD, Scherer PE. Adipocyte metabolism and obesity. J Lipid Res 2009;50(Suppl):S395-9.

[12] DeUgarte CM, Bartolucci AA, Azziz R, et al. Prevalence of insulin resistance in the polycystic ovary syndrome using the homeostasis model assessment. Fertil Steril 2005;83(5):1454-60.

[13] Teede HJ, Misso ML, Deeks AA, et al. Assessment and management of polycystic ovary syndrome: summary of an evidence-based guideline. Med J Aust 2011;195(6):S65-112.

[14] Kristensen S, Ramlau-Hansen CH, Ernst E, et al. A very large proportion of young Danish women have polycystic ovaries: is a revision of the Rotterdam criteria needed? Hum Reprod 2010;25(12):3117-22.

[15] Rotterdam ESHRE/ASRM-Sponsored PCOS consensus workshop group. Revised 2003 consensus on diagnostic criteria and long-term health risks related to polycystic ovary syndrome (PCOS). Hum Reprod 2004;19(1):41-7.

[16] Wang R, Mol BWJ. The Rotterdam criteria for polycystic ovary syndrome: evidence-based criteria? Human Reproduction 2017;32(2):261-4.

[17] Mohammad MB, Seghinsara AM. Polycystic Ovary Syndrome (PCOS), Diagnostic Criteria, and AMH. Asian Pac J Cancer Prev 2017;18(1):17-21.

[18] WHO Expert Consultation. Appropriate body-mass index for Asian populations and its implications for policy and intervention strategies. Lancet 2004;363(9403):157-63.

[19] Legro RS, Kunselman AR, Dodson WC, et al. Prevalence and predictors of risk for Type 2 diabetes mellitus and impaired glucose tolerance in polycystic ovary syndrome: a prospective controlled study in 254 affected women. J Clin Endocrinol Metab 1999;84(1):165-9.

[20] Aziz N, Kallur SD, Nirmalan PK. Implications of the revised consensus body mass indices for asian Indians on clinical obstetric practice. J Clin Diagn Res 2014;8(5):0C01-3.

[21] Jensen TK, Scheike T, Keiding N, et al. Fecundability in relation to body mass and menstrual cycle patterns. Epidemiology 1999;10(4):422-8.

[22] Lewis V. Polycystic ovary syndrome: a diagnostic challenge. Infertil Reprod Med Clin North Am 2003;14:497-516. 
[23] Aslan M, Celik O, Karsavuran N, et al. Maternal serum and cord blood preptin levels in gestational diabetes mellitus. J Perinatol 2011;31(5):350-5.

[24] Cohn GS, Kittleson MM, Blumenthal RS. Toward an improved diagnosis of the metabolic syndrome other clues to the presence of insulin resistance. Am J Hypertens 2005;18(8):1099-103.

[25] Gutch M, Kumar S, Razi SM, et al. Assessment of insulin sensitivity/resistance. Indian J Endocrinol Metab 2015;19(1):160-4.

[26] International evidence-based guideline for the assessment and management of Polycystic Ovary Syndrome (PCOS). https: / / www.monash.edu / medicine / sphpm / mchri / pcos / guideline.

[27] Gourgari E, Lodish M, Keil M, et al. Bilateral adrenal hyperplasia as a possible mechanism for hyperandrogenism in women with polycystic ovary syndrome. J Clin Endocrinol Metab 2016;101(9):3353-60.

[28] Teede HJ, Joham AE, Paul E, et al. Longitudinal weight gain in women identified with polycystic ovary syndrome: results of an observational study in young women. Obesity (Silver Spring) 2013;21(8):1526-32.

[29] Ganie MA, Kalra S. Polycystic ovary syndrome - a metabolic malady, the mother of all lifestyle disorders in women - can Indian health budget tackle it in future? Indian J Endocrinol Metab 2011;15(4):239-41.

[30] Randeva HS, Tan BK, Weickert MO, et al. Cardiometabolic aspects of the polycystic ovary syndrome. Endocr Rev 2012;33(5):812-41.
[31] Pericleous P, Stephanides S. Can resistance training improve the symptoms of polycystic ovary syndrome? BMJ Open Sport Exerc Med 2018;4(1):e000372.

[32] Cortés ME, Alfaro AA. The effects of hormonal contraceptives on glycemic regulation. Linacre $Q$ 2014;81(3):209-18.

[33] Jang C, Hui S, Lu W, et al. The small intestine converts dietary fructose into glucose and organic acids. Cell Metab 2018;27(2):351-361.e3.

[34] Pundir J, Psaroudakis D, Savnur P, et al. Inositol treatment of anovulation in women with polycystic ovary syndrome: a meta-analysis of randomised trials. Bjog 2018;125(3):299-308.

[35] Morais JBS, Severo JS, de Alencar GRR, et al. Effect of magnesium supplementation on insulin resistance in humans: a systematic review. Nutrition 2017;38:54-60.

[36] Harris HR, Titus LJ, Cramer DW, et al. Long and irregular menstrual cycles, polycystic ovary syndrome, and ovarian cancer risk in a population-based case-control study. Int J Cancer 2017;140(2):285-91.

[37] Rowland AS, Baird DD, Long S, et al. Influence of medical conditions and lifestyle factors on the menstrual cycle. Epidemiology 2002;13(6):668-74.

[38] Carroll JF, Kaiser KA, Franks SF, et al. Influence of BMI and gender on postprandial hormone responses. Obesity (Silver Spring) 2007;15(12):2974-83.

[39] Wang J, Yan R, Wen J, et al. Association of lower body mass index with increased glycemic variability in patients with newly diagnosed type 2 diabetes: a cross-sectional study in China. Oncotarget 2017;8(42):73133-43. 